\title{
Approximation by Complex Meyer-König and Zeller Operators
}

\author{
Qiulan Qi, Jianshuo Ma \\ School of Mathematical Sciences, Hebei Normal University, Shijiazhuang, China \\ Email:qiqiulan@163.com, majianshuo1008@126.com
}

How to cite this paper: Qi, Q.L. and Ma, J.S. (2020) Approximation by Complex Meyer-König and Zeller Operators. $A d$ vances in Pure Mathematics, 10, 1-11. https://doi.org/10.4236/apm.2020.101001

Received: November 25, 2019

Accepted: January 4, 2020

Published: January 7, 2020

Copyright $\odot 2020$ by author(s) and Scientific Research Publishing Inc. This work is licensed under the Creative Commons Attribution International License (CC BY 4.0).

http://creativecommons.org/licenses/by/4.0/

\begin{abstract}
The Meyer-König and Zeller operator is one of the most challenging operators. Sometimes the study of its properties will rely on the weighted approximation by Baskakov operator. In this paper, this relation is extended to complex space; the quantitative estimates and the Voronovskaja type results for analytic functions by complex Meyer-König and Zeller operators were obtained.
\end{abstract}

\section{Keywords}

Complex Meyer-König and Zeller Operators, Complex Baskakov Operators, Voronovskaja Type Result, Analytic Function

\section{Introduction}

The well known Meyer-König and Zeller operators are defined for functions $f(x) \in C[0,1)$ by [1]-[7]

$$
M_{n}(f, x)=\sum_{k=0}^{\infty} f\left(\frac{k}{n+k}\right) m_{n, k}(x),
$$

where $m_{n, k}(x)=\left(\begin{array}{c}n+k \\ k\end{array}\right) x^{k}(1-x)^{n+1}$.

The Meyer-König and Zeller operators [1]-[7], the Durrmeyer-type [8]-[15] have been the object of several investigations in approximation theory. The estimation of moments, the direct and inverse approximation properties were studied. Recently, many new modified types [12]-[19] have been constructed for different function spaces. Gal, Mahmudov, Opris etc. [16] [17] [18] [19] obtained the quantitative approximation estimates by complex Bernstein-type, Szász-type operators in compact disks.

The goal of this paper is to extend the results to complex Meyer-König and Zeller 
operators defined as follows: For analytic functions $f: \bar{D}_{R} \cup[R, 1) \rightarrow C, 0 \leq R<1$,

$$
M_{n}(f, z)=\sum_{k=0}^{\infty} f\left(\frac{k}{n+k}\right) m_{n, k}(z),
$$

where $m_{n, k}(z)=\left(\begin{array}{c}n+k \\ k\end{array}\right) z^{k}(1-z)^{n+1}, D_{R}=\{z \in C:|z|<R\}$.

We will obtain the following estimates for the complex Meyer-König and Zeller operators.

Theorem 1. Suppose that $f: \bar{D}_{R} \cup[R, 1) \rightarrow C$ is analytic in $\bar{D}_{R}$ and continuous in $[R, 1)$, that is, $f(z)=\sum_{p=0}^{\infty} c_{p} z^{p}$, for all $z \in \bar{D}_{R}$. Let $\sqrt{2}-1 \leq r<R<1$, for all $|z| \leq r$ and $n \geq 2$, we have

$$
\left|M_{n}(f, z)-f(z)\right| \leq \frac{M_{r}(f)}{n},
$$

where $M_{r}(f)=\sum_{p=1}^{\infty}\left|c_{p}\right|(2 p) ! r^{p-1}<+\infty$.

Theorem 2. Under the conditions of Theorem 1 , for all $|z| \leq r$ and $n \geq 2$, we have the following Voronovskaja type results

$$
\left|M_{n}(f, z)-f(z)-\frac{z}{2 n} f^{\prime \prime}(z)\right| \leq \frac{N_{r}(f)}{n^{2}},
$$

where

1) $N_{r}(f)=\sum_{p=1}^{\infty}\left|c_{p+1}\right| 5 p^{2}(2 p) ! r^{p-1}<+\infty$, for $\sqrt{2}-1 \leq r \leq \frac{\sqrt{5}-1}{2}$;

2) $N_{r}(f)=\sum_{p=1}^{\infty}\left|c_{p+1}\right| 5 p^{2}(2 p) ! r^{p+1}<+\infty$, for $\frac{\sqrt{5}-1}{2} \leq r<1$.

Theorem 3. Under the hypothesis of Theorem 2, if $f$ is not a polynomial of degree $\leq 1$ and the series $N_{r}(f)<+\infty$, then for $\sqrt{2}-1 \leq r<R<1$, we have

$$
\left\|M_{n}(f, z)-f(z)\right\|_{r} \sim \frac{1}{n}, n \in N,
$$

here $\|f\|_{r}=\sup \left\{|f(z)|: z \in D_{r}\right\}$.

The paper is organized as the following: In Section 2, we are going to promote the relationship between the Meyer-König and Zeller and Baskakov operators to complex space. In Section 3, we will study the approximation by the complex Baskakov operators. In Section 4, we will give the proof of Theorems 1 - 3. In Section 5, we will give the conclusion of this paper.

\section{The Connection between the Complex Meyer-König and Zeller and Baskakov Operators}

The proof is based on the connection between Meyer-König and Zeller and Baskakov operators. V. Totik was the first to use it [2] in the study of Meyer-König and Zeller operators, and many other afterward, see e.g. [6] [7] [8] [9] [11]. In this section, the connection will be extended to complex space. We will 
study a transformation $\tau$ mapping functions defined on $D_{l}=\left\{t \in C:\left|t-t_{0}\right|<l, 0 \leq l<+\infty\right.$, Ret $\left.>-\frac{1}{2}\right\}$ into functions defined on $D_{r}=\{z \in C:|z|<r, 0 \leq r<1\}$. The operator $\tau$ will allow us to relate the results for the complex Baskakov operators to their counterparts for the complex Meyer-König and Zeller operators. We will consider variables and functions defined on $D_{r}$ as $z, f(z)$ respectively, and their analogs defined on $D_{l}$, as the later will be denoted with $t, g(t)$. We consider the weight functions

$$
w_{1}(z)=w_{1}\left(\alpha_{0}, \alpha_{1}, z\right)=z^{\alpha_{0}}(1-z)^{\alpha_{1}}, z \neq 0,1, z \in D_{r}
$$

defined for real values of the parameters $\alpha_{0}, \alpha_{1} \in[-1,0]$. We will utilize the change $\sigma: D_{r} \rightarrow D_{l}$ given by

$$
t=\sigma(z)=\frac{z}{1-z}, z \in D_{r} .
$$

\section{Remark 1.}

$\sigma: D_{r}=\{z \in C:|z|<r, 0 \leq r<1\} \rightarrow D_{l}=\left\{t \in C:\left|t-t_{0}\right|<l, 0 \leq l<+\infty, \operatorname{Ret}>-\frac{1}{2}\right\}$, where $l=\frac{r}{1-r^{2}}, \quad t_{0}=\frac{r^{2}}{1-r^{2}}$. For example:

$\sigma: D_{\frac{1}{2}}=\left\{z \in C:|z|<\frac{1}{2}\right\} \rightarrow D_{\frac{2}{3}}=\left\{t \in C:\left|t-\frac{1}{3}\right|<\frac{2}{3}\right\}$.

Then, its inverse change $\sigma^{-1}: D_{l} \rightarrow D_{r}$ is

$$
z=\sigma^{-1}(t)=\frac{t}{1+t} \text {. }
$$

Remark 2. From the definition of $\sigma$ and $\sigma^{-1}$, we have that the change $\sigma$ and $\sigma^{-1}$ are linear fractional transformations and conformal mappings.

A function $g$ defined on $D_{l}$ is transformed to a function $f$ defined on $D_{r}$ by $\tau: g \rightarrow f$

$$
f(z)=\tau(g)(z)=\lambda(z)(g \circ \sigma)(z), \lambda(z)=1-z
$$

The inverse operator $\tau^{-1}$ transforming a function $f$ defined on $D_{r}$ to a function $g$ defined on $D_{l}$ is $\tau^{-1}: f \rightarrow g$

$$
g(t)=\tau^{-1}(f)(t)=\frac{1}{\left(\lambda \circ \sigma^{-1}\right)(t)}\left(f \circ \sigma^{-1}\right)(t), t \neq-1 .
$$

When a product of two functions is treated, that means, the associated operator $\Upsilon$ is defined by

$$
\Upsilon: w_{1}(z)=\Upsilon(w)(z)=\frac{1}{\lambda(z)}(w \circ \sigma)(z),
$$

and its inverse $\Upsilon^{-1}$ is defined by

$$
\Upsilon^{-1}: w(t)=\Upsilon^{-1}\left(w_{1}\right)(t)=\left(\lambda \circ \sigma^{-1}\right)(t)\left(w_{1} \circ \sigma^{-1}\right)(t) .
$$

For $f=\tau(g), w_{1}=\Upsilon(w)$, we have 


$$
\begin{gathered}
w_{1} f=\Upsilon(w) \tau(g)=(w \circ \sigma)(g \circ \sigma), \\
w g=\Upsilon^{-1}\left(w_{1}\right) \tau^{-1}(f)=\left(w_{1} \circ \sigma^{-1}\right)\left(f \circ \sigma^{-1}\right) .
\end{gathered}
$$

The operators $\tau$ and $\Upsilon$ have the following properties. From the definition (1)-(3), we yield immediately.

Proposition 1. Let $F_{r}, F_{l}$ denote the spaces of all functions defined on $D_{r}$ and $D_{l}$ respectively. Then $\tau: F_{l} \rightarrow F_{r}$ and $\tau^{-1}$ are linear operators.

Proposition 2. Let $w_{1}$ be a weight in $D_{r}, w=\Upsilon^{-1}\left(w_{1}\right)$,

$$
\begin{gathered}
F_{w_{1}}=\left\{f \in F_{r}: w_{1} f \in L_{\infty}\left(D_{r}\right)\right\} ; \\
F_{w}=\left\{g \in F_{l}: w g \in L_{\infty}\left(D_{l}\right)\right\} .
\end{gathered}
$$

Then the mapping $\tau: F_{w} \rightarrow F_{w_{1}}$ is a linear correspondence with $\left\|w_{1} \tau(g)\right\|_{r}=\|w g\|_{l},\left\|w \tau^{-1}(f)\right\|_{l}=\left\|w_{1} f\right\|_{r}$.

Proof. From the definition of the mapping $\tau$ (2) and the operator $\Upsilon$ (4), combining the Proposition 1, we get the mapping $\tau: F_{w} \rightarrow F_{w_{1}}$ is a linear correspondence.

Noting that the relation

$$
w_{1} \tau(g)(z)=\frac{1}{\lambda(z)}(w \circ \sigma)(z) \cdot \lambda(z)(g \circ \sigma)(z)=w(t) g(t),
$$

one can get the desired result.

The following proposition is very important, it gives the connection between the complex Meyer-König and Zeller operators and the complex Baskakov operators

$$
V_{n}(g, t)=\sum_{k=0}^{\infty} g\left(\frac{k}{n}\right) v_{n, k}(t),
$$

where $v_{n, k}(t)=\left(\begin{array}{c}n+k-1 \\ k\end{array}\right) t^{k}(1+t)^{-n-k}$.

Proposition 3. For every $f$ such that one of the series in (6) is convergent, for every $n \in N$, we have

$$
M_{n}(f, z)=\tau\left(V_{n}\left(\tau^{-1}(f)\right)\right)(z), z \in D_{r} .
$$

Proof. From the definition of the operator $V_{n}(g, t), M_{n}(f, z)$, Proposition 1 and the identities

$$
\begin{gathered}
\frac{n+k}{n} \tau\left(v_{n, k}\right)(z)=m_{n, k}(z), \\
\tau^{-1}(f)\left(\frac{k}{n}\right)=\frac{n+k}{n} f\left(\frac{k}{n+k}\right)
\end{gathered}
$$

valid for $k \in N \bigcup\{0\}$, we have (6).

Proposition 4. Under the conditions of Proposition 3, we have

$$
\left\|w_{1}\left(M_{n} f-f\right)\right\|_{r}=\left\|w\left(V_{n} g-g\right)\right\|_{l} .
$$

Proof. From Proposition 3, relations ((4), (1), (3)), we obtain for $g=\tau^{-1} f$ 
and $w=\Upsilon^{-1} w_{1}$,

$$
w_{1}\left(M_{n} f-f\right)=\left(w\left(V_{n} g-g\right)\right) \circ \sigma
$$

and hence

$$
\left\|w_{1}\left(M_{n} f-f\right)\right\|_{r}=\left\|w\left(V_{n} g-g\right)\right\|_{l} .
$$

Remark 3. If the weight $w_{1}(z)=1$ (i.e. $\alpha_{0}=\alpha_{1}=0$ ), the corresponding weight to $w_{1}(z)=1$ is $w(t)=\frac{1}{1+t}$.

Then, we have the following auxiliary results.

Lemma 2.1. Under the conditions of Proposition 3, $w_{1}(z)=1, w(t)=\frac{1}{1+t}$, we have

$$
\left\|M_{n} f-f\right\|_{r}=\left\|w(t)\left(V_{n} g-g\right)\right\|_{l} .
$$

Lemma 2.2. [16] Denoting $e_{p}(t)=t^{p}$ and $T_{n, p}(t)=V_{n}\left(e_{p}, t\right), T_{n, p}(t)$ is a polynomial of degree $p, \quad p=0,1,2, \cdots$, we have the recurrence formula

$$
T_{n, p+1}(t)=\frac{t(t+1)}{n} T_{n, p}^{\prime}(t)+t T_{n, p}(t) .
$$

\section{Weighted Approximation by the Complex Baskakov Operators}

Theorems 1 - 3 will be proved in Section 4 by transferring the corresponding results for the complex Baskakov operators. In this section, we will prove some properties of the complex Baskakov operators. The first main result of this section is the following theorem for upper bound.

Theorem 3.1. Suppose that $g: \bar{D}_{L} \cup[L,+\infty) \rightarrow C$ is continuous in $\bar{D}_{L} \cup[L,+\infty)$ and analytic in $\bar{D}_{L}$, i.e. $g(t)=\sum_{p=0}^{\infty} c_{p} t^{p}$. Let $\frac{1}{2} \leq l<L<+\infty$, for all $|t| \leq l, n \geq 2$, we have

$$
\left\|w(t)\left(V_{n}(g, t)-g(t)\right)\right\|_{l} \leq \frac{M_{l}(g)}{n},
$$

where $M_{l}(g)=\sum_{p=1}^{\infty}\left|c_{p}\right|(2 p) ! l^{p-1}<+\infty, w(t)=\frac{1}{1+t}$.

Proof. By using the recurrence relation of Lemma 2.2, for all $t \in C, p=0,1,2, \cdots, n \geq 2$, we have

$$
T_{n, p+1}(t)=\frac{t(t+1)}{n} T_{n, p}^{\prime}(t)+t T_{n, p}(t) .
$$

From this we immediately get the recurrence formula

$$
w(t)\left(T_{n, p}(t)-t^{p}\right)=\frac{t}{n}\left(T_{n, p-1}(t)-t^{p-1}\right)^{\prime}+t\left[w(t)\left(T_{n, p-1}(t)-t^{p-1}\right)\right]+\frac{p-1}{n} t^{p-1} .
$$

To estimate $\left\|w(t)\left(T_{n, p}(t)-e_{p}(t)\right)\right\|_{l}$, we wil use the relation [16] p. 7: 
$\left|B_{k}^{\prime}(t)\right| \leq \frac{k}{l}\left\|B_{k}\right\|_{l}$ for all $|t| \leq l$, where $B_{k}(t)$ is a polynomial of degree $\leq k$.

Then, we get

$$
\begin{aligned}
& \left\|w(t)\left(T_{n, p}(t)-e_{p}(t)\right)\right\|_{l} \\
& \leq \frac{l}{n}\left\|T_{n, p-1}(t)-e_{p-1}(t)\right\|_{l} \frac{p-1}{l}+l\left\|w(t)\left(T_{n, p-1}(t)-e_{p-1}(t)\right)\right\|_{l}+\frac{p-1}{n} l^{p-1},
\end{aligned}
$$

which implies

$$
\begin{aligned}
& \left\|w(t)\left(T_{n, p}(t)-e_{p}(t)\right)\right\|_{l} \\
& \leq\left(\frac{3 l(p-1)}{n}+l\right)\left\|w(t)\left(T_{n, p-1}(t)-e_{p-1}(t)\right)\right\|_{l}+\frac{p-1}{n} l^{p-1} .
\end{aligned}
$$

We will prove the following relation by mathematical induction with respect to $p$ :

$$
\left\|w(t)\left(T_{n, p}(t)-e_{p}(t)\right)\right\|_{l} \leq \frac{(2 p) !}{n} l^{p-1}
$$

Indeed for $p=1,\left\|w(t)\left(T_{n, 1}(t)-e_{1}(t)\right)\right\|_{l}=0 \leq \frac{2}{n}$. Suppose that it is true for $p>1$, that is,

$$
\left\|w(t)\left(T_{n, p}(t)-e_{p}(t)\right)\right\|_{l} \leq \frac{(2 p) !}{n} l^{p-1} .
$$

Now for $p+1$, by the relations $((7),(8))$, we have

$$
\left\|w(t)\left(T_{n, p+1}(t)-e_{p+1}(t)\right)\right\|_{l} \leq\left(\frac{3 l p}{n}+l\right) \frac{(2 p) !}{n} l^{p-1}+\frac{p}{n} l^{p} .
$$

It remains to prove that for $n \geq 2$

$$
\left(\frac{3 l p}{n}+l\right) \frac{(2 p) !}{n} l^{p-1}+\frac{p}{n} l^{p} \leq \frac{(2(p+1)) !}{n} l^{p} .
$$

By mathematical induction that the last inequality holds true for all $p \geq 1$ and $n \geq 2$. From the hypothesis on $g$, it follows that $V_{n}(g, t)$ is analytic in $D_{l}$, we write

$$
\left\|w(t)\left(V_{n}(g, t)-g(t)\right)\right\|_{l} \leq \sum_{p=1}^{\infty}\left|c_{p}\right| \cdot\left\|w(t)\left(T_{n, p}(t)-e_{p}(t)\right)\right\|_{l} \leq \sum_{p=1}^{\infty}\left|c_{p}\right| \frac{(2 p) !}{n} l^{p-1} .
$$

Theorem 3.2. Under the conditions of Theorem 3.1, let $\frac{1}{2} \leq l<L<+\infty$, for all $|t| \leq l, n \geq 2$, we have the following Voronovskaja type formula

$$
\left|w(t)\left(V_{n}(g, t)-g(t)-\frac{t(1+t)}{2 n} g^{\prime \prime}(t)\right)\right| \leq \frac{N_{l}(g)}{n^{2}},
$$

where

$$
\text { 1) for } \frac{1}{2} \leq l<L<1, \quad N_{l}(g)=\sum_{p=1}^{\infty}\left|c_{p+1}\right| 5 p^{2}(2 p) ! l^{p-1}<+\infty \text {; }
$$


2) for $1 \leq l<L<+\infty, \quad N_{l}(g)=\sum_{p=1}^{\infty}\left|c_{p+1}\right| 5 p^{2}(2 p) ! l^{p+1}<+\infty$.

Proof. Case I. For $\frac{1}{2} \leq l<L<1$, noting that $e_{p}(t)=t^{p}, p=0,1,2, \cdots$ and $T_{n, p}(t)=V_{n}\left(e_{p}, t\right)$ and $V_{n}(g, t)=\sum_{p=0}^{\infty} c_{p} V_{n}\left(e_{p}, t\right)$, we have

$$
\begin{aligned}
& \left|w(t)\left(V_{n}(g, t)-g(t)-\frac{t(1+t)}{2 n} g^{\prime \prime}(t)\right)\right| \\
& \leq \sum_{p=1}^{\infty}\left|c_{p}\right| \mid w(t)\left(T_{n, p}(t)-e_{p}(t)-\frac{p(p-1)(1+t)}{2 n} t^{p-1}\right) .
\end{aligned}
$$

Using the recurrence relation of Lemma 2.2, we write

$$
T_{n, p+1}(t)=\frac{t(t+1)}{n} T_{n, p}^{\prime}(t)+t T_{n, p}(t) .
$$

Denote that

$$
E_{n, p}(t)=T_{n, p}(t)-e_{p}(t)-\frac{p(p-1)(1+t)}{2 n} t^{p-1} .
$$

Noting that $T_{n, 1}(t)-e_{1}(t)=0$, for $p \geq 2$, we have

$E_{n, p}^{\prime}(t)=\frac{n}{t(1+t)} T_{n, p+1}(t)-\frac{n}{1+t} T_{n, p}(t)-p t^{p-1}-\frac{p^{2}(p-1)}{2 n} t^{p-1}-\frac{p(p-1)^{2}}{2 n} t^{p-2}$.

By simple computation, we get

$E_{n, p+1}(t)=\frac{t(1+t)}{n} E_{n, p}^{\prime}(t)+t E_{n, p}(t)+\frac{p^{2}(p-1)(1+t)}{2 n^{2}} t^{p}+\frac{p(p-1)^{2}(1+t)}{2 n^{2}} t^{p-1}$.

Thus, for all $p, n \in N,|t|<l, \frac{1}{2} \leq l<L<1$, we have

$$
\left|w(t) E_{n, p+1}(t)\right| \leq \frac{l(1+l)}{n}\left|w(t) E_{n, p}^{\prime}(t)\right|+l\left|w(t) E_{n, p}(t)\right|+\frac{2 p^{3}}{n^{2}} l^{p-1} .
$$

Using the estimate in the proof of Theorem 3.1, for all $p \in N, n \geq 2$ and $\frac{1}{2} \leq l<L<1$, we have

$$
\left\|w(t)\left(T_{n, p}(t)-e_{p}(t)\right)\right\|_{l} \leq \frac{(2 p) !}{n} l^{p-1} .
$$

Now we shall estimate $\left|w(t) E_{n, p}^{\prime}(t)\right|$ for $p \geq 2$. Noting that $E_{n, p}(t)$ is a polynomial of degree $\leq p$, combining the Bernstein's inequality, we have

$$
\begin{aligned}
\left|w(t) E_{n, p}^{\prime}(t)\right| & \leq \frac{p}{l}\left\|w(t) E_{n, p}(t)\right\|_{l} \\
& \leq \frac{p}{l}\left[\left\|w(t)\left(T_{n, p}(t)-e_{p}(t)\right)\right\|_{l}+\left\|w(t) \frac{p(p-1)(1+t)}{n} t^{p-1}\right\|_{l}\right] \\
& \leq \frac{p}{l}\left(\frac{(2 p) !}{n} l^{p-1}+\frac{p^{2}}{n} l^{p-1}\right) \leq \frac{2 p(2 p) !}{n} l^{p-2},
\end{aligned}
$$


thus,

$$
\begin{gathered}
\frac{l(1+l)}{n}\left|w(t) E_{n, p}^{\prime}(t)\right| \leq \frac{4 p(2 p) !}{n^{2}} l^{p-1}, \\
\left|w(t) E_{n, p+1}(t)\right| \leq \frac{4 p(2 p) !}{n^{2}} l^{p-1}+l\left|w(t) E_{n, p}(t)\right|+\frac{2 p^{3}}{n^{2}} l^{p-1}, \\
\left|w(t) E_{n, p+1}(t)\right| \leq \frac{5 p(2 p) !}{n^{2}} l^{p-1}+l\left|w(t) E_{n, p}(t)\right|,
\end{gathered}
$$

we obtain step by step following

$$
\left|w(t) E_{n, p+1}(t)\right| \leq \frac{5 p l^{p-1}}{n^{2}} \sum_{j=1}^{p}(2 j) ! \leq \frac{5 p^{2}(2 p) !}{n^{2}} l^{p-1},
$$

which follows that

$$
\left|w(t)\left(V_{n}(g, t)-g(t)-\frac{t(1+t)}{2 n} g^{\prime \prime}(t)\right)\right| \leq \frac{N_{l}(g)}{n^{2}},
$$

where $N_{l}(g)=\sum_{p=2}^{\infty}\left|c_{p}\right| 5(p-1)^{2}[2(p-1)] ! I^{p-2}<+\infty$.

Case 2. For $1 \leq l<L$, in the proof of Case 1, the relation (9) should be changed to

$$
\left|w(t) E_{n, p+1}(t)\right| \leq \frac{l(1+l)}{n}\left|w(t) E_{n, p}^{\prime}(t)\right|+l\left|w(t) E_{n, p}(t)\right|+\frac{2 p^{3}}{n^{2}} l^{p+1},
$$

and the relation (10) should be changed to

$$
\begin{aligned}
\left|w(t) E_{n, p+1}(t)\right| & \leq \frac{4 p(2 p) !}{n^{2}} l^{p-1}+l\left|w(t) E_{n, p}(t)\right|+\frac{2 p^{3}}{n^{2}} l^{p+1} \\
& \leq \frac{5 p(2 p) !}{n^{2}} l^{p+1}+l\left|w(t) E_{n, p}(t)\right|,
\end{aligned}
$$

then,

$$
N_{l}(g)=\sum_{p=1}^{\infty}\left|c_{p+1}\right| 5 p^{2}(2 p) ! l^{p+1}<+\infty
$$

\section{The Proof of Theorems 1 - 3}

The Proof of Theorem 1. Combining Lemma 2.1 and Theorem 3.1, we can obtain Theorem 1.

The Proof of Theorem 2. From Lemma 2.1 and Theorem 3.2, we have Theorem 2 .

In what follows we obtain the exact degree in the approximation by $M_{n}(f, z)$.

Theorem 4.1. Suppose that the hypothesis on the function $f$ and Theorem 2. If $f$ is not a polynomial of degree $\leq 1$ and the series $N_{r}(f)<+\infty$, then $\left\|M_{n} f-f\right\|_{r} \geq \frac{C_{r}(f)}{n}$ holds, where $C_{r}(f)$ depends only on $f$ and $r$.

Proof. For all $|z| \leq r$, we can write 


$$
M_{n}(f, z)-f(z)=\frac{1}{n}\left[z f^{\prime \prime}(z)+\frac{1}{n} n^{2}\left(M_{n}(f, z)-f(z)-\frac{z}{n} f^{\prime \prime}(z)\right)\right] .
$$

Applying the inequality $\|F+G\|_{r} \geq\|F\|_{r}-\|G\|_{r} \mid \geq\|F\|_{r}-\|G\|_{r}$, we obtain

$$
\left\|M_{n}(f, z)-f(z)\right\|_{r} \geq \frac{1}{n}\left[\left\|z f^{\prime \prime}(z)\right\|_{r}-\frac{1}{n} n^{2}\left\|\left(M_{n}(f, z)-f(z)-\frac{z}{n} f^{\prime \prime}(z)\right)\right\|_{r}\right] .
$$

Since $f$ is not a polynomial of degree $\leq 1$ in $D_{R}$, we get $\left\|z f^{\prime \prime}(z)\right\|_{r}>0$. Indeed, supposing the contrary, it follows that $z f^{\prime \prime}(z)=0$ for all $|z| \leq r$, which implies $f^{\prime \prime}(z)=0$ for all $z \in \bar{D}_{R} \backslash\{0\}$. Since $f$ is analytic in $\bar{D}_{R}$, this means that $f^{\prime \prime}(z)=0$ for all $z \in \bar{D}_{R}$, that is $f$ is a polynomial of degree $\leq 1$, a contradiction with the hypothesis.

Now by Theorem 2, for $N_{r}(f)<+\infty$, we have

$$
n\left|M_{n}(f, z)-f(z)-\frac{z}{2 n} f^{\prime \prime}(z)\right| \leq \frac{N_{r}(f)}{n} \rightarrow 0(n \rightarrow \infty) .
$$

Choose $n_{1}$, such that for all $n \geq n_{1}$, we have

$$
\left\|z f^{\prime \prime}(z)\right\|_{r}-\left\|n\left(M_{n}(f, z)-f(z)-\frac{z}{n} f^{\prime \prime}\right)(z)\right\|_{r} \geq \frac{1}{2}\left\|z f^{\prime \prime}(z)\right\|_{r},
$$

which implies for all $n \geq n_{1}$,

$$
\left\|M_{n}(f, z)-f(z)\right\|_{r} \geq \frac{1}{2 n}\left\|z f^{\prime \prime}(z)\right\|_{r} .
$$

For $1 \leq n \leq n_{1}-1$, we have

$$
\left\|M_{n}(f, z)-f(z)\right\|_{r} \geq \frac{1}{n} n\left\|M_{n}(f, z)-f(z)\right\|_{r}=\frac{C_{n, r}(f)}{n}>0,
$$

i.e. $\left\|M_{n}(f, z)-f(z)\right\|_{r} \geq \frac{C_{r}(f)}{n}$, here

$$
C_{r}(f)=\min \left\{C_{1, r}(f), C_{2, r}(f), \cdots, C_{n_{1}-1, r}(f), \frac{1}{2}\left\|z f^{\prime \prime}(z)\right\|_{r}\right\} .
$$

The Proof of Theorem 3. From Lemma 2.1, Theorem 4.1 and Theorem 1, we can obtain Theorem 3.

\section{Conclusion}

In this paper, the properties of approximation are studied by using the general relation between the Meyer-König and Zeller and Baskakov operators. The geometric properties (the shap-preserving) of such complex operators still remain to be studied.

\section{Acknowledgements}

We thank the Editor and the referee for their comments. The work is partially supported by NSF of China $(11571089,11871191)$ and NSF of Hebei Province (2012205028; ZD2019053). The project supported by science foundation of He- 
bei Normal University.

\section{Conflicts of Interest}

The authors declare no conflicts of interest regarding the publication of this paper.

\section{References}

[1] Becker, M. and Nessel, R.J. (1978) A Global Approximation Theorem for Meyer-König and Zeller Operators. Mathematische Zeitschrift, 160, 195-206.

https://doi.org/10.1007/BF01237033

[2] Totik, V. (1983) Uniform Approximation by Baskakov and Meyer-König and Zeller Operators. Periodica Mathematica Hungarica, 14, 209-228. https://doi.org/10.1007/BF01849019

[3] Abel, U. (1995) The Moments for the Meyer-König and Zeller Operators. Journal of Approximation Theory, 82, 352-361. https://doi.org/10.1006/jath.1995.1084

[4] Chen, W. (1986) On the Integral Type Meyer-König and Zeller Operators. Approximation Theory and Its Applications, 2, 7-18.

[5] Guo, S.S. and Qi, Q.L. (2007) The Moments for the Meyer-König and Zeller Operators. Applied Mathematics Letters, 20, 719-722. https://doi.org/10.1016/j.aml.2006.09.002

[6] Gadjev, I. (2015) Strong Converse Result for Uniform Approximation by Meyer-König and Zeller Operators. Journal of Mathematical Analysis and Applications, 428, 32-42. https://doi.org/10.1016/j.jmaa.2015.03.004

[7] Ivanov, K.G. and Parvanov, P.E. (2012) Weighted Approximation by Meyer-König and Zeller Operators. In: Nikolov, G. and Uluchev, R., Eds., Constructive Theory of Functions, Sozopol 2010, Academic Publishing House, Sofia, 150-160.

[8] Heilmann, M. (2003) Commutativity of Durrmeyer-Type Modifications of Meyer-König and Zeller and Baskakov Operators. In: Bojanov, B.D., Ed., Constructive Theory of Functions, Varna 2002, Darba, Sofia, 295-301.

[9] Heilmann, M. (2003) Eigenfunctions of Durrmeyer-Type Modifications of Meyer-König and Zeller and Baskakov Operators. Journal of Approximation Theory, 125, 63-73. https://doi.org/10.1016/j.jat.2003.09.006

[10] Abel, U., Gupta, V. and Ivav, M. (2004) The Complete Asymptotic Expansion for a General Durrmeyer Variant of the Meyer-König and Zeller Operators. Mathematical and Computer Modelling, 40, 867-875. https://doi.org/10.1016/j.mcm.2004.10.016

[11] Heilmann, M. (2006) Eigenfunctions and Eigenvalues for Some Durrmeyer-Type Operators. In: Bojanov, B.D., Ed., Constructive Theory of Functions, Varna 2005, Academic Publishing House, Sofia, 158-167.

[12] Harun, K. (2017) Approximation by Urysohn Type Meyer-König and Zeller Operators to Urysohn Integral Operators. Results in Mathematics, 72, 1571-1583. https://doi.org/10.1007/s00025-017-0729-x

[13] Mehmet, A. (2016) New Korovkin Type Theorem for Non-Tensor Meyer-König and Zeller Operators. Results in Mathematics, 69, 327-343. https://doi.org/10.1007/s00025-015-0472-0

[14] Holhos, A. (2018) Weighted Approximation of Functions by Meyer-König and Zeller Operators of Max-Product Type. Numerical Functional Analysis and Optimiza- 
tion, 39, 689-703. https://doi.org/10.1080/01630563.2017.1413386

[15] Zhou, G. and Cai, Q. (2019) Triangular Surface Patch Based on Bivariate Meyer-König and Zeller Operators. Open Mathematics, 17, 282-296. https://doi.org/10.1515/math-2019-0021

[16] Gal, S.G. (2009) Approximation by Complex Bernstein and Convolution Type Operators. World Scientific Publ. Co., Singapore, Hong Kong, London. https://doi.org/10.1142/7426

[17] Gal, S.G. (2008) Approximation and Geometric Properties of Complex FavardSzász-Mirakjan Operators in Compact Disks. Computers \& Mathematics with Applications, 56, 1121-1127. https://doi.org/10.1016/j.camwa.2008.02.014

[18] Gal, S.G. and Gupta, V. (2014) Approximation by Complex Szász-Durrmeyer Operators in Compact Disks. Acta Mathematica Scientia, 34B, 1157-1165. https://doi.org/10.1016/S0252-9602(14)60076-X

[19] Gal, S.G., Mahmudov, N.I. and Opris, B.D. (2016) Approximation with an Arbitrary Order by Szász, Szász-Kantorovich and Baskakov Complex Operators in Compact Disks. Azerbaijan Journal of Mathematics, 6, 3-12. 